\title{
On the Classification of Toric Fano Varieties
}

\section{Günter Ewald}

Mathematisches Institut, Ruhr.Universität Bochum, Universitätstrasse 150, NA2173,

D.4630 Bochum, Federal Republic of Germany

\begin{abstract}
Toric Fano varieties are algebraic varieties associated with a special class of convex polytopes in $R^{\prime \prime}$. We extend results of V. E. Voskresenskij and A. A. Klyachko about the classification of such varieties using a purely combinatorial method of proof.
\end{abstract}

Let $P$ be a simplical convex polytope in $\mathbb{R}^{n}$ whose vertices are primitive lattice vectors $\left(\in \mathbb{Z}^{n}\right)$, and which contains 0 in its interior. If $a_{1}, \ldots, a_{n}$ are the vertices of a facet of $P$ we suppose $\operatorname{det}\left(a_{1}, \ldots, a_{n}\right)= \pm 1$, for all facets of $P$. Then we call $P$ a Fano polytope.

Let $\Sigma=\Sigma(P)$ be a system of cones each of which joins 0 to all points of a face of $P$, so that the toric or toroidal variety $X_{\Sigma}$ associated with the fan $\Sigma$ (see, for example, [1], [2], [3]) is projective. In case $P$ is a Fano polytope, $X_{\Sigma(P)}$ is said to be a toric (or toroidal) Fano variety. (It is, equivalently, a complete smooth toric variety whose anticanonical divisor is ample.)

Let $e_{1}, \ldots, e_{n}$ be the canonical basis vectors of $\mathbb{R}^{n}$. If $n=2 k$ is even $>0$; then for $Q:=\operatorname{conv}\left\{ \pm e_{1}, \ldots, \pm e_{n}, e_{1}-e_{2}+\cdots+e_{n-1}-e_{n},-e_{1}+e_{2}+\cdots+e_{n-1}-e_{n}\right\}$ we obtain an example of a Fano variety $X_{\Sigma(Q)}$, called a del Pezzo variety $V^{2 k}$. It can be obtained from $\mathbb{P}^{1} \times \cdots \times \mathbb{P}^{1}$ ( $n$ times) by blowing-up twice in regular points. Voskresenskij and Klyachko [4] have classified all symmetric toroidal Fano varieties, i.e., Fano varieties $X_{\Sigma(P)}$ with centrally symmetric $P$, hence possessing a torus-invariant symmetry:

Any symmetric toroidal Fano variety splits into a product of projective

lines and del Pezzo varieties.

In terms of convexity a split $X_{\Sigma(P)}=X_{1} \times X_{2}$ of $X_{\Sigma(P)}$ is given by $P=\operatorname{conv}\left(P_{1} \cup P_{2}\right)$ where $P_{1}, P_{2}$ are polytopes whose linear spans have only 0 in common. So $X_{\Sigma(P)}=X_{\Sigma\left(P_{1}\right)} \times X_{\Sigma\left(P_{2}\right)}$. A dual polytope $P^{*}$ of $P$ then decomposes into the 
Minkowski sum $P^{*}=P_{1}^{*}+P_{2}^{*}$ where $P_{1}^{*}, P_{2}^{*}$ are dual lattice polytopes of $P_{1}, P_{2}$ relative to aff $P_{1}$, aff $P_{2}$ (affine hulls), respectively, such that $\operatorname{dim}\left(\right.$ aff $\left.P_{1} \cap P_{2}\right)=0$. This again implies that the invertible sheaf associated with $P^{*}$ splits into the tensor product of the invertible sheafs associated with $P_{1}^{*}, P_{2}^{*}$. If $X_{\Sigma(P)}$ does not split we call $P$ irreducible.

In their proof of (1) the authors make use of an interesting relationship between symmetric Fano varieties and Dynkin diagrams of root systems. Nevertheless, in this note we present a direct and short proof of (1). Furthermore, we extend the result as follows:

We call a Fano polytope pseudo-symmetric if it has two facets $F, F^{\prime}$ centrally symmetric to each other with respect to 0 . Let, for example, $n$ be even and

$$
Q:=\operatorname{conv}\left\{ \pm e_{1}, \ldots, \pm e_{n}, e_{1}-e_{2}+\cdots+e_{n-1}-e_{n}\right\}
$$

In this case we call $X_{\Sigma(Q)}$ a pseudo-del Pezzo variety. We shall prove:

Theorem. Any pseudo-symmetric toroidal Fano variety splits into a product of projective lines, del Pezzo varieties, and pseudo-del Pezzo varieties.

Some consequences may be noted:

Any pseudo symmetric Fano variety

$$
X_{\Sigma}=\mathbb{P}_{1}^{(1)} \times \cdots \times \mathbb{P}_{1}^{(P)} \times V^{2 k_{1}} \times \cdots \times V^{2 k_{r}} \times \tilde{V}^{2 m_{1}} \times \cdots \times \tilde{V}^{2 m_{s}}
$$

$\left(\mathbb{P}_{1}^{(q)}\right.$ projective lines, $V^{2 k_{\imath}}$ del Pezzo varieties, $\tilde{V}^{2 m_{J}}$ pseudo-del Pezzo varieties) can be blown-down

(a) into a product $\mathbb{P}_{1}^{(1)} \times \cdots \times \mathbb{P}^{(n)}$ of projective lines; $2 r+s(\leq n)$ blow-downs are hereby needed;

(b) into a product $\mathbb{P}_{1}^{(1)} \times \cdots \times \mathbb{P}_{1}^{(\rho)} \times \mathbb{P}_{2 k_{1}} \times \cdots \times \mathbb{P}_{2 k_{r}} \times \mathbb{P}_{2 m_{1}} \times \cdots \times \mathbb{P}_{2 m}$ of projective spaces; $r+k_{1}+\cdots+k_{r}+m_{1}+\cdots+m_{s}(\leq n+r)$ blow-downs are hereby needed.

We say, a polytope $P$ is inscribed in a polytope $\hat{P}$ if $\operatorname{dim} P=\operatorname{dim} \hat{P}$, and if any vertex of $P$ is also a vertex of $\hat{P}$. We call a Fano polytope maximal if it is not inscribed in any Fano polytope $\hat{P} \neq P$.

Each maximal pseudo-symmetric Fano polytope is centrally symmetric.

Further Fano polytopes can be obtained from the pseudo-symmetric ones by omitting vertices and considering the convex hull of the remaining polytopes (provided, it contains 0 in its interior). The following polytope $P \subset \mathbb{R}^{3}$, however, is not of this type:

$$
P=\operatorname{conv}\left\{e_{1}, e_{2}, e_{3},-e_{2},-e_{3},-e_{1}-e_{2},-e_{2}+e_{3}\right\} .
$$


A construction method ("suspension") for Fano polytopes is also presented by Voskresenskij and Klyachko [4] using Gale diagrams. Since this construction assigns to any lattice polytope $P_{0}$ a Fano polytope $P$ the authors doubt whether it might make sense to try a classification of all Fano polytopes. It should be noted, however, that the suspension (in [2] also used for the construction of nonprojective toroidal varieties) destroys the original structure of $P$. Also, the authors show that the number of vertices of any Fano polytope in $\mathbb{R}^{n}$ is $\leq n^{2}+1$. So we think a classification is possible. As intermediate results we conjecture:

Conjecture 1. There are at most $n-1$ types of maximal irreducible $n$-dimensional Fano polytopes. Each of them possesses at most $2 n+2$ vertices.

Conjecture 2. Up to a unimodular transformation, all vertices of a Fano polytope have coordinates $1,-1,0$ only.

The proof of our theorem is achieved by proving several lemmas.

Lemma 1. Let the coordinates be chosen such that $F=\operatorname{conv}\left\{e_{1}, e_{2}, \ldots, e_{n}\right\}$ and $F^{\prime}=\operatorname{conv}\left\{-e_{1}, \ldots,-e_{n}\right\}$ are facets of a pseudo-symmetric Fano polytope $P$. Then any further vertex $a=\left(a_{1}, \ldots, a_{n}\right)$ of $P$ satisfies

$$
-1 \leq a_{j} \leq 1, \quad j=1, \ldots, n .
$$

Proof. Let (for row vectors)

$$
\delta=\left(\begin{array}{cccc}
1 & 0 & \cdots & 0 \\
1 & 1 & & \vdots \\
\vdots & & \ddots & 0 \\
1 & 0 & \cdots & 1
\end{array}\right)
$$

be a unimodular transformation. Then

$$
\begin{gathered}
F^{\delta}=\operatorname{conv}\left\{e_{1}, e_{1}+e_{2}, \ldots, e_{1}+e_{n}\right\} \\
F^{\prime \delta}=\operatorname{conv}\left\{-e_{1},-e_{1}-e_{2}, \ldots,-e_{1}-e_{n}\right\}
\end{gathered}
$$

Let

$$
F_{j}:=\operatorname{conv}\left\{e_{1}, e_{1}+e_{2}, \ldots, e_{1}+e_{j-1}, e_{1}+e_{j+1}, \ldots, e_{1}+e_{n}\right\}
$$

be a face of $F^{\delta}$, and let $F_{a}:=\operatorname{conv}\left(\{a\} \cup F_{j}\right)$ be a facet of $P^{\delta}$ adjacent to $F^{\delta}$. Clearly, $\left|a_{1}\right|<1$, hence $a_{1}=0$. From the regularity of $X_{\Sigma(P)}$ we obtain

$$
a_{j}=\operatorname{det}\left(e_{1}, e_{1}+e_{2}, \ldots, e_{1}+e_{j-1}, a, e_{1}+e_{j+1}, \ldots, e_{1}+e_{n}\right)=-1 .
$$

The supporting hyperplane $H_{j}:=\left\{x_{1}-x_{j}=1\right\}$ of $P^{\delta}$ carried by $F_{a}$ intersects the $x_{j}$-axis in $\left(0, \ldots, 0, t_{j}, 0, \ldots, 0\right)$ where $-1 \leq t,<0$. Furthermore, $H_{j} \cap\left\{x_{1}=0\right\}$ is a 
supporting hyperplane of $P^{\delta} \cap\left\{x_{1}=0\right\}$ relative to $\left\{x_{1}=0\right\}$. Since all vertices $\notin$ $F^{\delta} \cup F^{\prime \delta}$ of $P^{\delta}$ lie in $\left\{x_{1}=0\right\},\left\{x_{j}=-1\right\}$ is a supporting hyperplane of $P^{\delta}, j=$ $2, \ldots, n$. Similarly, we see that $\left\{x_{j}=+1\right\}$ is a supporting hyperplane of $P^{\delta}$, $j=2, \ldots, n$.

If $\hat{F}:=\operatorname{conv}\left\{b, e_{1}+e_{2}, \ldots, e_{1}+e_{n}\right\}$ is a facet adjacent to $F$ we obtain, from the regularity of $X_{\Sigma}$,

$$
-b_{2}-\cdots-b_{n}=\operatorname{det}\left(b, e_{1}+e_{2}, \ldots, e_{1}+e_{n}\right)=-1 .
$$

The supporting hyperplane carried by $\hat{F}$ has the equation $x_{2}+\cdots+x_{n}=1$. Also $x_{2}+\cdots+x_{n}=-1$ is a supporting hyperplane of $P^{\delta}$. Therefore,

$$
-1 \leq x_{2}+\cdots+x_{n} \leq 1 \text { for all vertices } x=\left(x_{1}, \ldots, x_{n}\right) \text { of } P^{\delta} \text {. }
$$

Now $\left(0, x_{2}, \ldots, x_{n}\right)^{\delta^{-1}}=\left(-x_{2}-\cdots-x_{n}, x_{2}, \ldots, x_{n}\right) ;$ furthermore, the hyperplanes $\left\{x_{j}=1\right\}$ and $\left\{x_{j}=-1\right\}, j=2, \ldots, n$, are invariant under $\delta^{-1}$. So all vertices of $P$ satisfy (4).

Lemma 2. Let $a=\left(a_{1}, \ldots, a_{n}\right), a^{\prime}=\left(a_{1}^{\prime}, \ldots, a_{n}^{\prime}\right)$ be two vertices $\neq \pm e_{1}, \ldots, \pm e_{n}$ of $P$. Then for any $j=1, \ldots, n$, neither $a_{j}=a_{j}^{\prime}=1$ nor $a_{j}=a_{j}^{\prime}-1$ is true.

Proof. If $j>1$ we can, according to the proof of Lemma 1 , consider $P^{\delta}$ instead of $P$. Then $a_{1}=a_{1}^{\prime}=0$ and we deduce from $a_{j}=a_{j}^{\prime}=-1$ that both, $a$ and $a^{\prime}$, lie in the supporting hyperplane $H_{j}$ of $P$. This implies that $e_{1}^{\delta}, \ldots, e_{j-1}^{\delta}, e_{j+1}^{\delta}, \ldots, e_{n}^{\delta}$, $a, a^{\prime}$ are affinely dependent, a contradiction, since $P^{\delta}$ is simplicial.

If $j=1$ we may assume $n>1$ (otherwise everything is trivial), and interchange the roles of 1 and $a j_{0}>1$.

We call vertices $\neq \pm e_{1}, \ldots, \pm e_{n}$ of $P$ extra vertices.

Lemma 3. Any extra vertex a of $P$ lies in the hyperplane $x_{1}+\cdots+x_{n}=0$ and hence can be represented (up to renumbering of coordinates) in the form

$$
a=(1,-1, \ldots, 1,-1,0, \ldots, 0) \quad(\text { possibly no } 0) \text {. }
$$

Proof. This follows from the fact that $F, F^{\prime}$ carry the supporting hyperplanes $x_{1}+\cdots+x_{n}=1, x_{1}+\cdots+x_{n}=-1$, respectively.

Proof of (1). If $P$ is centrally symmetric with respect to 0 , and if $a=$ $(1,-1, \ldots, 1,-1,0, \ldots, 0)$ is an extra vertex, then also $-a$ is an extra vertex. By Lemma 3 the matrix having all extra vertices as rows splits as follows:

$$
\left(\begin{array}{rrrrr|r}
1 & -1 & \cdots & 1 & -1 & 0 \\
-1 & 1 & \cdots & -1 & 1 & \\
\hline & 0 & & & *
\end{array}\right)
$$

This implies $P$ itself splits unless $a$ and $-a$ have no zero coordinates. 
Lemma 4. If $P$ does not split, $n=\operatorname{dim} P>1$, then $n$ is even and either $a=$ $(1,-1, \ldots, 1,-1)$ is the only extra vertex or $a,-a$ are the only extra vertices.

Proof. Suppose, Lemma 4 is false and we have two extra vertices $a, b$. Then we can assume the matrix of extra vertices to have the form ( $m$ always even)

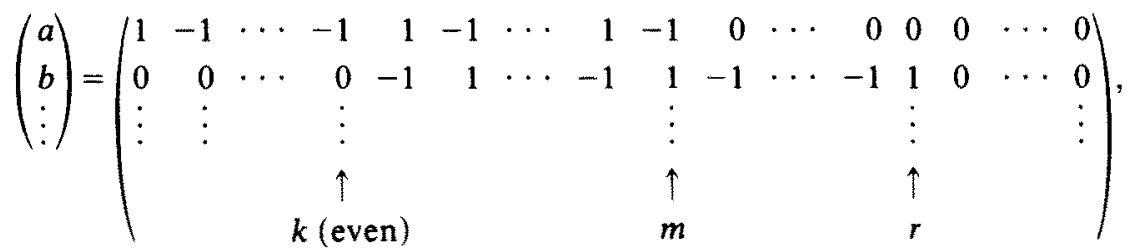

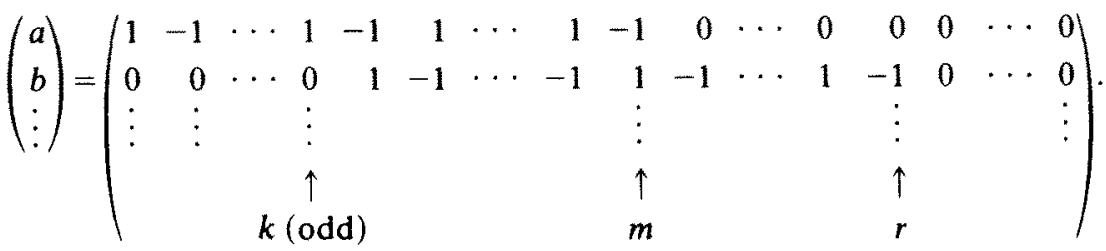

In both cases at least one 0 occurs in the first row; furthermore, $k<m-1$. Setting $e_{r-1}^{z}:=-e_{r-1}$ we obtain in case $(5)$

$$
a+b-e_{1}+e_{2}-\cdots-e_{k-1}+e_{k}+e_{m+1}-e_{m+2}-\cdots-e_{r-2}-e_{r-1}^{\prime}-e_{r}=0
$$

which expresses an affine dependence of $e_{1}, \ldots, e_{k}, e_{m+1}, \ldots, e_{r-2}, e_{r-1}^{\prime}, e_{r}, a, b$. These points lie in the hyperplane

$$
L(x):=x_{1}+\cdots+x_{k-1}+x_{k}+x_{k+1}+x_{m+1}+\cdots+x_{r-2}-x_{r-1}+x_{r}=1 .
$$

For all vertices $\pm e_{j}$ we have $L\left( \pm e_{j}\right) \leq 1$. If $c$ is a further extra vertex we can write the matrix of extra vertices as

$$
\begin{aligned}
& \left(\begin{array}{c}
a \\
b \\
c \\
\vdots
\end{array}\right)=\left(\begin{array}{rrlrrrrrrrr}
1 & -1 & \cdots & 1 & -1 & 1 & \cdots & -1 & 1 & -1 & \cdots \\
0 & 0 & \cdots & 0 & 0 & 0 & \cdots & 0 & -1 & 1 & \cdots \\
-1 & 1 & \cdots & -1 & 1 & 0 & \cdots & 0 & 0 & 0 & \cdots \\
\vdots & \vdots & & & & & & \vdots & & & \\
& & & & & & & \uparrow & & & \\
& & & & & & & k & & &
\end{array}\right. \\
& \left.\begin{array}{rrrrlrrrrrrrr}
1 & -1 & 0 & 0 & \cdots & 0 & 0 & 0 & \cdots & 0 & 0 & \cdots & 0 \\
-1 & 1 & -1 & 1 & \cdots & -1 & 1 & -1 & \cdots & 1 & 0 & \cdots & 0 \\
0 & 0 & 1 & -1 & \cdots & 1 & -1 & 0 & \cdots & 0 & 0 & \cdots & 0 \\
& \vdots & & & & & & & & \vdots & & & \vdots \\
& \uparrow & & & & & & & & \uparrow & & & \\
& m & & & & & & & & & & &
\end{array}\right)
\end{aligned}
$$


from which we see $L(c) \leq 1$. Hence $L(x)=1$ is a supporting hyperplane of $P$. this implies $e_{1}, \ldots, e_{k}, e_{m+1}, \ldots, e_{r-2}, e_{r-1}^{\prime}, e_{r}, a, b$ to be vertices of a nonsimplicial face of $P$, a contradiction.

Suppose now case (6). We obtain (for $e_{r}^{\prime}:=-e_{r}$ )

$$
a+b-e_{1}+e_{2}-\cdots-e_{k}+e_{m+1}-e_{m+2}+\cdots+e_{r-2}-e_{r-1}-e_{r}^{\prime}=0,
$$

which is an affine dependence of the points involved. These points lie on the hyperplane

$$
L(x):=x_{1}+x_{2}+\cdots+x_{k}+x_{m+1}+\cdots+x_{r-1}-x_{r}=1
$$

Again it is seen that $L\left( \pm e_{j}\right) \leq 1, j=1, \ldots, n$, also $L(c) \leq 1$ for any other vertex $c$ of $P$. This implies again a contradiction to $P$ being simplicial.

Therefore, no 0 occurs in $a$ and $b$, from which the lemma follows.

From Lemmas 1-4 the theorem is now readily obtained.

\section{References}

1. V. Danilov, the geometry of toric varieties, Uspekhi Mat. Nauk 33 (1978), 85-134. Russian Math. Surveys 33 (1978), 97-154.

2. G. Ewald, Spherical complexes and nonprojective toric varieties, Discrefe Comput. Geom. 1 (1986), $115-122$

3. T. Oda, Torus Embeddings and Applications, Tata Institute, Bombay, 1978.

4. V. E. Voskresenskij and A. A. Klyachko, Toroidal Fano varieties and root systems. Izv. Nauk 48 (1984), Math. USSR.Izv. 24 (1985), 221-244. 